\title{
Evaluation of External Dose Equivalent with Thermoluminescent Dosimeters from Residents Living in Radiation-contaminated Buildings
}

\author{
J. S. LEE*1, S. L. DONG ${ }^{1}$, W. P. CHANG ${ }^{2}$ and C. C. CHAN ${ }^{3}$ \\ ${ }^{1}$ Division of Radiological Science and Technology, National Yang-Ming University, 155 Li-Nong St., \\ Sec. \#2, Taipei 112, Taiwan, 'Institute of Public Health, National Yang-Ming University, 155 \\ Li-Nong St., Sec. \#2, Taipei 112, Taiwan and 'Institute of Public Health, National Taiwan \\ University, Taipei, Taiwan
}

(Received 30 December 1996; in revised form 24 April 1997)

\begin{abstract}
As of October 1996 there are more than 90 radiation-contaminated steel supported rebar buildings (containing more than 1000 apartments) dispersed in the northern part of Taiwan. These apartments were contaminated with cobalt 60 at a total activity ranging from $1-140 \mu \mathrm{Sv} / \mathrm{yr}$. In this paper, a method is developed for evaluating external dose equivalent and dose equivalent rates encountered by the residents wearing specially designed thermoluminescent dosimeter (TLD)-embedded chains, belts and badges. Comparisons are also made between the TLD readings and the exposure readings from indoor layout personal dosimetry surveys and room occupancy adjustments to the buildings. The accuracy and sensitivity of the TLDs compared with the ionization chamber readings are judged to be considerable improvements over those of previous studies. From the present study, it is concluded that the reliability of the daily activity records provided by the residents during the entire TLD-wearing period is the most critical but challenging feature of the external dose equivalent measurement. (C) 1997 Elsevier Science Ltd. All rights reserved
\end{abstract}

\section{Introduction}

Since the initiation of the Atomic Bomb Survivors (ABS) study in Japan, there have been many epidemiological studies indicating increased health risks, particularly risks in induced cancer, of acute high-dose exposure (BEIR-III, 1980; BEIR-V, 1990; ICRP, 60, ; ICRU, 47, ). The dose effects are not relevant for chronic low-dose exposure, however controversies have persisted through a number of similar studies (Maruyama et al., 1988; Cardis et al., 1995). Limited information on occupational or living experiences around nuclear power or reprocessing plants reveals further conflicting results (Lubenau and Nussbaumer, 1986; Gilbert, 1991; Cardis et al., 1995).

As of October 1996 there are more than 90 radiation-contaminated rebar buildings (containing more than 1000 apartments) scattered in the northern regions of Taiwan. These apartments were contaminated with cobalt-60 at a total activity ranging

*To whom all correspondence should be addressed. from 1-140 mSv/yr (Chang and Chang, 1996). The primary sources of the ${ }^{60} \mathrm{Co}$ radionuclide contamination were from the recycling of discharged radioactive steel produced in one of the major steel reprocessing factories in Taiwan, and the contaminated steel rods were then used for construction of rebar buildings (Marley, 1993; Chang and Kau, 1993; Lubenau and Yusko, 1995; Cardarelli II et al., 1997). Although these are highly controversial and potentially drastic environmental health problems, comprehensive radiation dose reconstructions from these contaminated buildings and residents can provide the best opportunity for a meaningful epidemiological study and can offer a more profound interpretation of the impact of such a radiation hazard on the population.

In the framework of dose reconstruction issues, there are two major complicating factors involved in the evaluation of external dose equivalents experienced by the residents. One is that the exposure rates among the contaminated apartments have varied greatly from location to location and room to room depending on the levels of contamination of the steel 
rods and geometrical indoor layout differences. The other is that most of the residents who have lived in the apartments have moved out precipitously and at different times, making the retrospective dose measurements difficult to assess (Maruyama et al., 1988; Haskell, 1993; Degteva et al., 1994, 1996; Goksu et al., 1996). For these reasons, it appears impractical to perform complete area surveys in each building. However, it should be possible to make area surveys of the selected buildings in order to develop dose assessment models that are easy to operate and relatively accurate. One comprehensive study conducted by the Atomic Energy Council (AEC) of Taiwan has used a $\mu \mathrm{R}$ ionization chamber and thermoluminescent dosimeters (TLDs) for the building sites survey and cumulative exposure measurements (Chang and Chang, 1996). Another was developed by the National Institute for Occupational and Safety Health (NIOSH), U.S.A., applying the indoor layouts of the buildings and parameter corrections (such as energy spectrum, time decay, geometric and shielding effect, indoor activities, etc.) to establish a retrospective dose assessment model (Cardarelli II et al., 1997). From these models, the cumulative doses to the residents and buildings can then be determined. Even with these adjustment and correction factors, both studies have either not fully taken into account daily activity patterns, height adjustments of the residents, or not considered sensitivity of TLDs, physical indoor layouts of the buildings, therefore the external dose equivalents may be potentially overestimated.

To obtain a quantitative estimate of the total stochastic risks, it is essential to know the mean dose equivalent to a set of organs and tissues. The most effective way to approach this is to convince the residents living in the contaminated buildings to wear TLD badges for a period of time, and the TLD signals can then be correlated further with the doses from retrospective models to test their efficacy (Gilbert, 1991; Gilbert et al., 1996; Degteva et al., 1996).

In the present paper, a method is developed for evaluating external dose equivalents from the residents wearing specially designed TLD-embedded chains, belts and badges. Comparisons are also made with the exposure readings from indoor layout surveys and room occupancy adjustments to the buildings. The strong and weak points of the approach are evaluated and discussed.

\section{Materials and Methods}

\section{Radiation area survey}

There are more than 90 radiation-contaminated rebar buildings (containing more than 1000 apartments) dispersed in the northern part of Taiwan. These buildings are subgrouped as high, medium and low radiation regions according to the levels of contamination. We have selected five buildings from each subgroup for radiation area surveys. The completed area survey is conducted with two types of ionization chambers (AD series and Bicron $\mu$-analyst) and the survey area includes walls, ceilings, closets, living rooms, bedrooms, kitchen, dining room and bathrooms. A typical indoor layout of an apartment and the dose equivalent rate readings in the rooms are given in Fig. 1. In the apartment, five exposure rate readings with equally divided distance in each room are measured. With the height differences of the family members and dose distributions to the body in mind, at each location another five readings at the heights of the thyroid, chest, abdomen, gonad and knee regions are also taken. The average from the five equally spaced readings is then assigned to each room as average room dose rate at each body location. For those frequently occupied areas, such as dining table, sofas, and beds, three extra readings at each location are also measured.

\section{LiF-100H TLD dose response study}

To test the sensitivity and linearity of the TLDs, a complete absorbed dose response study is conducted. We have chosen LiF-100H (LiF:Mg,Cu,P) TLD disks as dosimeter for all the dose measurements. First, a pack of 200 TLD disks is annealed at $240^{\circ} \mathrm{C}$ for $10 \mathrm{~min}$ as recommended by the vendor and then each TLD disk receives a dose of $1 \mathrm{cGy}$ of ${ }^{60} \mathrm{Co}$ gamma-radiation. The TLD disks are read with a Harshaw 4000 TLD reader, and the reading charge signals are corrected to obtain calibration factors for the TLD disks. Second, in the dose response study the TLD disks are further subdivided into 36 subgroups and accordingly receive doses ranging from 0 to $3 \mathrm{cGy}$ of ${ }^{60} \mathrm{Co}$ irradiation. The whole regression relationship between the TLD reading signals and the given doses reflects the sensitivity and degree of linearity of the TLD disks to gamma-ray response.

In surveying the contaminated buildings, because the ionization chamber readings fluctuate greatly from $0.1 \mu \mathrm{Sv} / \mathrm{h}$ to $1 \mathrm{mSv} / \mathrm{h}$ and can only provide approximate space radiation levels at a given time, we have placed an extra 50 TLDs at specific locations in the apartments for one week for intercomparison purposes.

\section{External TLD dose measurements from the residents}

For the TLD dose measurement, residents still living in the contaminated apartments are contacted and asked to wear specially designed TLD-embedded chains, belts and badges for about one to four weeks, depending on the levels of contamination in the apartments. During the wearing period, the residents are instructed to keep their daily activity patterns as usual as possible, and the head of a family is instructed to record a daily activity history for each 


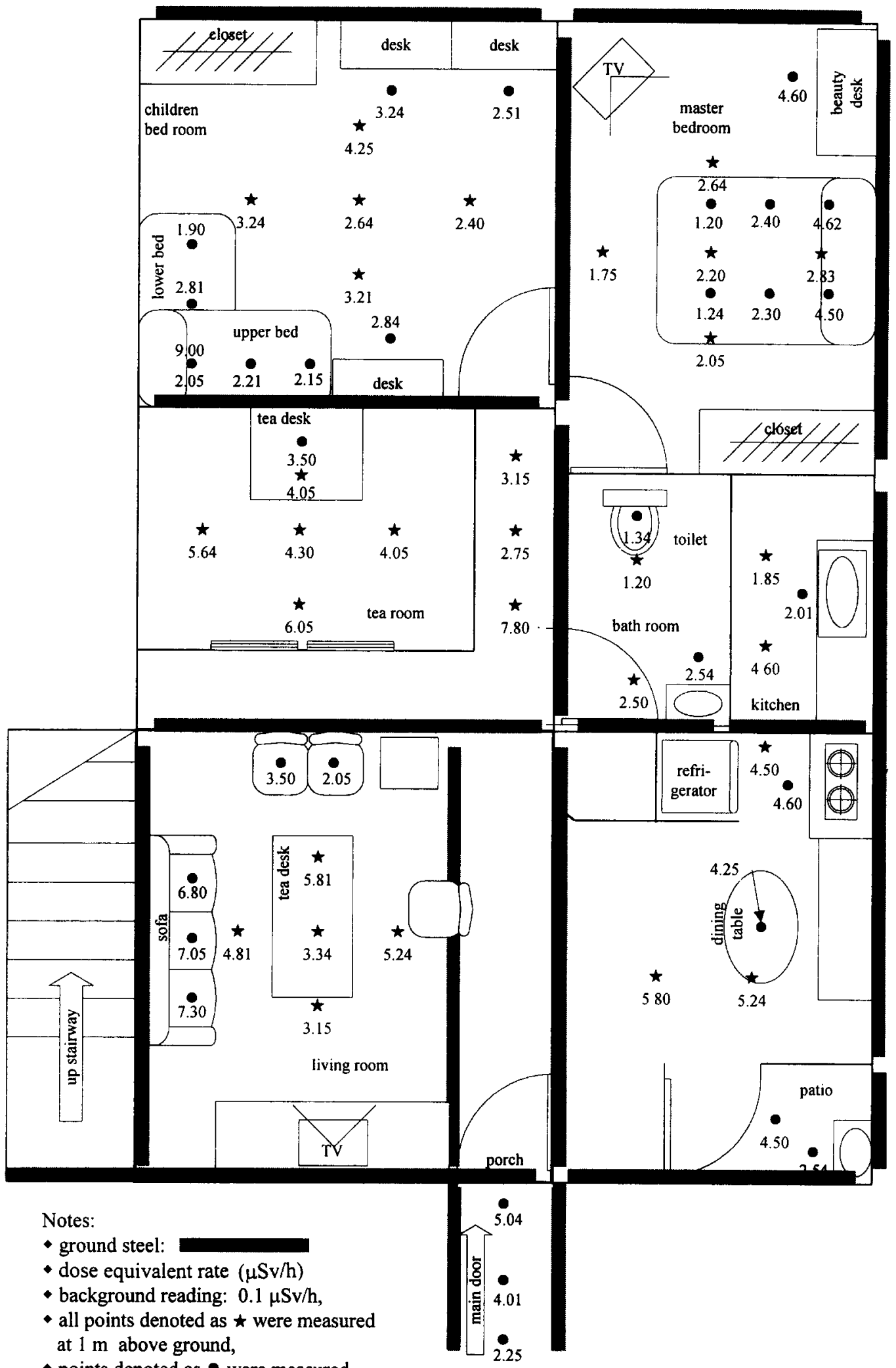

- points denoted as $\bullet$ were measured at their corresponding sites.

Fig. 1. A typical apartment indoor layout and dose equivalent rates evaluated at specific locations. In each room, five readings are taken and the average of the readings is assigned to the room as average dose equivalent rate. For those frequently occupied areas, such as dining table, sofas and beds, three extra readings at each location are also measured. 


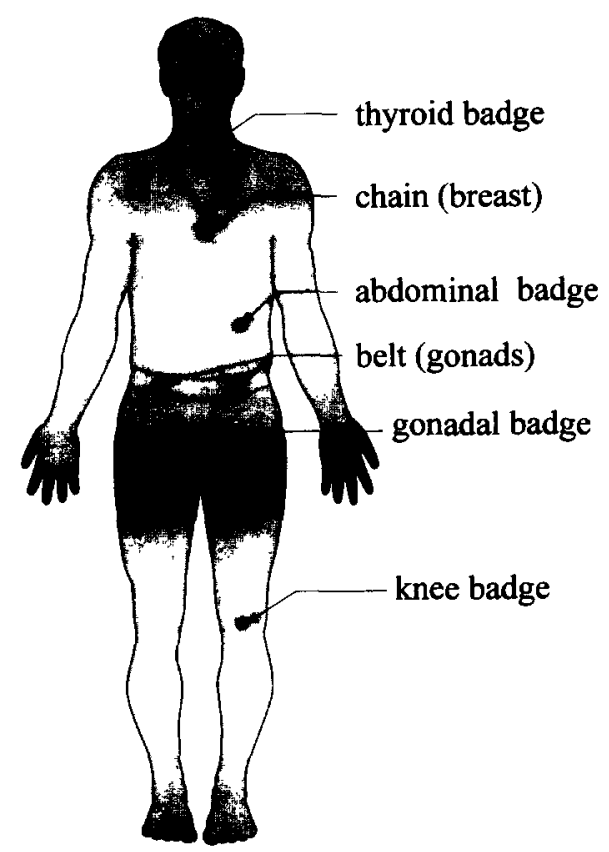

Fig. 2. Body locations of the TLD-embedded chain, belt and badges used for external dose measurements. The sites of interest for measurement are the thyroid, breast, abdominal. gonadal and knee areas.

individual in the family. For each individual, two TLDs are inserted in the chain to simulate the doses to breast area, and the other four TLDs are placed in the belt (two are at anterior-posterior [AP] positions, and the other two are at lateral positions) to simulate doses to abdominal organs. Two extra TLD badges are placed at thyroid and gonadal areas, respectively. The TLD locations for chain, belt and four badge locations are shown in Fig. 2.

Adjustment factor $(A F)$ and room occupancy factor (rof)

The relationship between the ionization chamber readings and the TLD measurements forms an adjustment factor (Chang and Chang, 1996; Cardarelli II et al., 1997). The factor represents the effectiveness of the ionization chamber in determining the dose equivalent to the residents and is defined as

\section{Adjustment factor (AF)}

$$
\begin{aligned}
& \text { Dose equivalent obtained from } \\
& \text { the TLD disks } H \text { (TLD) } \\
& =\overline{\text { Dose equivalent obtained from }} \\
& \text { the ionization chamber } H(\mathrm{R})
\end{aligned}
$$

To determine exactly the external dose equivalents to the residents, we need to know how much time the residents spend in each room of the apartment. Based on the daily activity histories provided by the family, one can determine the room occupancy factor (rof) (Cardarelli II et al., 1997), which accounts for the percentage of room occupation by each individual, and is given as

room occupancy factor (rof)

$$
=\frac{\text { hours of a room used by the resident }}{24 \text { hours (whole day) }}
$$

The height differences among the family members may greatly affect the TLD dose interpretations. In particular, doses from the adults may not be the same as children's, since radiation angles directed to different parts of the body are different. It is then necessary to apply height adjustments to each individual to reflect actual dose distribution in the body. The procedure for height adjustment applied to the family members is illustrated in Table 1. For example, the dose at the thyroid of a young adult can be matched to the average dose from the thyroid and chest areas of an adult, and for children the dose at the abdomen approximately equals the dose at the gonadal area of an adult.

Finally, the external dose equivalent to the specific organ of the residents can be determined as follows:

External dose equivalent $\left(\bar{H}_{\mathrm{ex}}\right)_{t}$

$$
=\left[\bar{H}(\mathrm{R}), \times \mathrm{AF}_{l} \times \operatorname{rof}_{l} \times h\right] i=\mathbf{t}, \mathbf{c}, \mathbf{a}, \mathbf{g}, \mathbf{k}
$$

where $\bar{H}(\mathrm{R})$, is the average room dose rate measured at height of organ $i, \mathrm{AF}$ is the adjustment factor, rof is the room occupancy factor, $h$ is the total hours

Table 1. The dose assignment procedure for height adjustment to the individual family members. For example, for a young adult, the dose at the thyroid is about the same as the average dose from thyroid and chest areas of an adult, and for children, the dose at the abdomen approximately equals the dose at the gonadal area

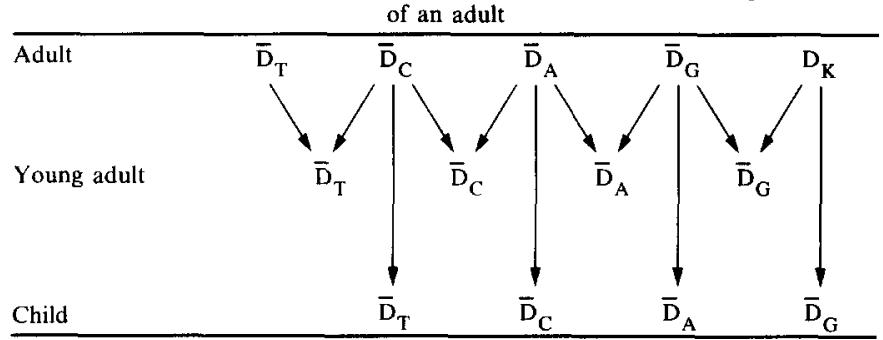

$\mathrm{T}=$ thyroid: $\mathrm{C}=$ chest; $\mathrm{A}=$ abdomen; $\mathrm{G}=$ gonads; $\mathrm{K}=$ knee 


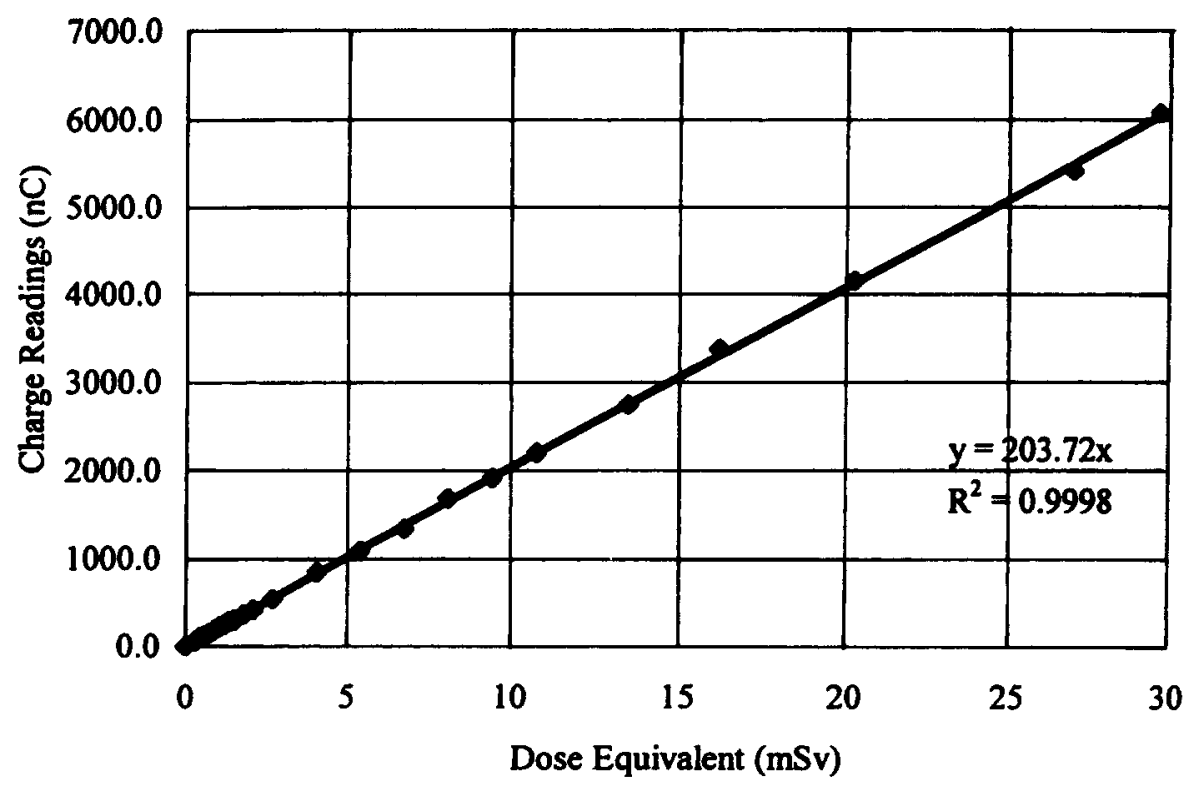

Fig. 3. The linearity and dose-response characteristics of LiF-100H TLD. The dose equivalent ranging from $0-30 \mathrm{mSv}$ shows a direct linear relationship with the collected charge signals.

spent in a room by the resident, $\mathrm{t}, \mathrm{c}, \mathrm{a}, \mathrm{g}, \mathrm{k}$ are the thyroid, chest, abdomen, gonad and knee, respectively.

\section{Results and Discussion}

The dose linearity of the response characteristics of the LiF-100H TLDs is verified by the results in Fig. 3, in terms of a linear relationship between the collected charge signals and dose equivalent in the range of $0-30 \mathrm{mSv}$. In comparing the ionization chamber readings with the TLD measurements, the corresponding adjustment factors (AF) are given in Table 2. These adjustment factors suggest that the ionization chamber has much better sensitivity in measuring radiation signals and should still be considered the primary choice for area surveys. The adjustment factors in our study show far better agreement (approximately 0.9) than the value of 0.67 used by the NIOSH model (Cardarelli II et al., 1997). This better agreement may be due to our LiF-100 TLD having higher sensitivity than that of the one reported by the NIOSH in terms of doses to the

Table 2. The adjustment factors" (AF) between ionization chamber and TLD readings for different family members and different body locations. The average value of the AFs is 0.91 , which shows better agreement than the value of 0.67 used by the NIOSH model

\begin{tabular}{lcccc}
\hline & Thyroid & Chest & Abdomen & Gonads \\
\hline Husband & $b$ & 0.93 & 0.81 & 0.96 \\
Housewife & 0.93 & 0.81 & 0.89 & 0.93 \\
Child 1 & 0.87 & 0.94 & 0.93 & 0.99 \\
Child 2 & 0.89 & 0.95 & 0.90 & 0.89 \\
Mean & 0.90 & 0.91 & 0.88 & 0.94 \\
\hline
\end{tabular}

-Adjustment factors (AF) $=$ TLD readings/ionization chamber readings.

'The husband did not wear a thyroid badge. residents and the shorter badge wearing period (one week in our study is much shorter than the one month used in the NIOSH model), which potentially makes our daily activity records more reliable. Moreover, the factors suggest how effective measurements by the ionization chamber may be for making dose calculations in terms of individual external dose measurements. They also indicate the fact that the sensitivity and linearity of the TLDs essentially determine how long the chains, belts and badges should be worn. For highly contaminated buildings, where exposure rates are greater than $15 \mu \mathrm{Sv} / \mathrm{h}$, one week for TLD wearing period is usually sufficient.

In the area surveys, we found that at least three readings for each location are necessary to gain acceptable accuracy for the rooms, and, if the room size is large (such as a living room), then more readings may be required. For those considered as highly occupied areas (such as kitchen, dining table, sofa, bed and bathrooms), five readings are suggested to secure accurate doses to the residents.

The daily activity record provided by the residents during the entire TLD wearing period is the most important but also a difficult task for external dose measurement. The daily activity needs to be faithfully and cooperatively recorded by the residents. Basically, getting strong coordination from one member of a family is extremely important. Another significant difficulty is that the daily activity patterns of the residents may have changed after they were notified as residents living in the contaminated buildings. The residents tend to avoid the radiation sources, and the changes would tend to make the TLD measurements underestimations of the dose equivalent values (Rosenstein, 1982; Gilbert 
Table 3. An example of the weighted dose equivalent rate calculation for an individual with the room occupancy factors

\begin{tabular}{|c|c|c|c|c|c|}
\hline & \multirow{2}{*}{$\begin{array}{l}\text { Room occupancy } \\
\text { factor (rof) }\end{array}$} & \multicolumn{4}{|c|}{ Dose equivalent rate (DER) $(\mu \mathrm{Sv} / \mathrm{h})$} \\
\hline & & Thyroid & Chest & Abdomen & Gonads \\
\hline Living room & 0.1495 & $2.95 \pm 0.15$ & $2.80 \pm 0.05$ & $2.65 \pm 0.20$ & $2.15 \pm 0.05$ \\
\hline Dining room & 0.0074 & $2.45 \pm 0.20$ & $2.20 \pm 0.10$ & $2.20 \pm 0.05$ & $1.65 \pm 0.15$ \\
\hline Bedroom & 0.3240 & $2.70 \pm 0.10$ & $2.60 \pm 0.12$ & $1.80 \pm 0.20$ & $1.50 \pm 0.05$ \\
\hline Bathroom & 0.0453 & $1.00 \pm 0.10$ & $1.20 \pm 0.05$ & $1.30 \pm 0.05$ & $1.30 \pm 0.05$ \\
\hline Kitchen & 0.0052 & $1.20 \pm 0.10$ & $1.40 \pm 0.10$ & $2.85 \pm 0.15$ & $4.55 \pm 0.15$ \\
\hline Study desk & 0.0638 & $1.85 \pm 0.05$ & $1.90 \pm 0.10$ & $2.05 \pm 0.10$ & $2.25 \pm 0.15$ \\
\hline Weighted dose equivalent rate $(\mu \mathrm{Sv} / \mathrm{h})^{\mathrm{c}}$ & & $1.50 \pm 0.07$ & $1.46 \pm 0.06$ & $1.20 \pm 0.11$ & $1.05 \pm 0.04$ \\
\hline
\end{tabular}

"Dose calculation for a housewife in a contaminated bulding.

${ }^{b}$ Room occupancy factor (rof) $=$ resident hours in each room $/ 24$ hours.

'Weighted DER for a specific organ $=\Sigma$, (rof,$\times$ DER of a specific organ at room $i)$.

et al., 1996). However, it would be very difficult to justify their discrepancies during the entire measurement. The room occupancy factors (rof) of each room are therefore determined from the daily activity records and applied to the weighted dose equivalent calculations. An example of calculation with these factors applied to the residents is given in Table 3.

For a given measurement, there is a marked gradient in the TLD dose equivalent distribution from thyroid to gonads, as seen in Table 4. These results indicate that the radiation was primarily coming from the top direction. However, the dose distributions for the adults may not necessarily be the same as for the children. In our research, a comprehensive directional (AP, top-bottom and rotational) dependence study was not performed, as the radiation actually may come from all directions. Moreover, it may not be convenient for all residents to wear TLD badges at all locations on the body for more than one week. The dose calculation may therefore be somewhat limited due to geometrical factors not being fully taken into account (Grosswendt and Hohlfeld, 1989; Gilbert et al., 1996).

The height adjustment for young adults and children is also necessary, because radiation doses to adults may not be the same as those to children as radiation angles contributed to the body are different. Although the assignment for height adjustments to young adults and children is based purely on judgment, it would reflect realistic dose distributions to family members.

\section{Conclusions}

The history of daily activities faithfully recorded by the residents is the most crucial feature in the external dose measurement. From our experience, a limitation in the measurement is the difficulty in convincing the residents comprehensively to wear the multiple TLD badges for more than one week. The longer the wearing period, the more likely the residents will be to lose patience and the records, therefore, become less dependable.

The average dose rates among the contaminated buildings were from $1-140 \mu \mathrm{Sv} / \mathrm{h}$, which may be considered an excellent chronic low-dose exposure model. Such a model also confirms that the sensitivity and linearity of the response of the TLDs is an important prerequisite for getting accurate dose assessments. With features of approximate tissue equivalence, relatively less susceptibility to environmental extremes, quick and easy anneal preparation, high sensitivity and accuracy, the LiF-100H has proven to be the best choice for the study. The accuracy of dose rate readings in the buildings is sufficient for external dose equivalent calculations. For those rooms frequently occupied (i.e. rof close to 1) by the residents, a suitable number of readings is needed to ensure that accurate mean dose rates can be obtained for calculations.

Most dosimetry systems, including those used in our study, are usually calibrated only at AP geometry, that is under the assumption that the TLD dosimeter is worn on the front of the torso, and that exposure is directed from the front to the back. However, in the contaminated buildings, radiation comes from all directions and the TLD dosimeters were not worn in the optimum position. This would ultimately result in the measured doses being somewhat skewed (Xu et al., 1995; Gilbert et al., 1996). To obtain the necessary information for geometricalcorrection, it may be necessary to conduct laboratory simulations by investigating the relationship between

Table 4. An example of annual equivalent dose distributions ${ }^{\star}$ for typical family members; the doses are for an eight-day period of wearing TLD badges

\begin{tabular}{lccrc}
\hline & Thyroid & \multicolumn{1}{c}{ Chest } & \multicolumn{1}{c}{ Abdomen } & Gonads \\
\hline Husband & $\mathrm{b}$ & $11.63 \pm 0.14$ & $7.53 \pm 0.02$ & $7.18 \pm 0.01$ \\
Housewife & $13.14 \pm 0.69$ & $12.79 \pm 0.23$ & $10.51 \pm 0.22$ & $9.20 \pm 0.37$ \\
Child 1c & $8.26 \pm 0.61$ & $8.10 \pm 0.23$ & $6.69 \pm 0.32$ & $6.74 \pm 0.03$ \\
Child 2c & $9.01 \pm 0.30$ & $9.16 \pm 0.17$ & $8.33 \pm 0.14$ & $8.71 \pm 0.05$ \\
\hline
\end{tabular}

"Annual accumulated dose equivalent $(\mathrm{mSv} / \mathrm{yr})$ for a specific organ $=$ [weighted dose equivalent rate $(\mu \mathrm{Sv} / \mathrm{h}) \times \mathrm{AF}$ ] of a specific organ $\times 365$ (day/year) $\times 24$ (hour/day) $/ 1000$.

'The husband did not wear a thyroid badge.

'Child 1 is 12 yrs old and child 2 is 9 yrs old. 
doses delivered at several angles of incidence, which will be the next goal of our study.

For the purpose of comparing risk estimates, it is organ doses that are of greatest interest (Rosenstein, 1982; Schultz and Zoetelief, 1996). Converting external dose to doses in various organs is usually available in the literature, which provides many tables of conversion factors (Fisher and Snyder, 1967; BEIR-III, 1980; BEIR-V, 1990; ICRU, 39, ICRU, 43, ICRU, 47). These relationships, which depend strongly on both energy and geometry, are based on computer simulations using the spherical and anthropomorphic (Alderson Rando) phantoms. However, in our case, radiation is essentially isotropic in the buildings, which is usually not the situation stated in the literature, and it is inappropriate to assign uncertainty factors for the entire experiment. A complete simulation with the Rando phantom (Rosenstein, 1982; Golikov and Nikitin, 1989) in the contaminated buildings, based on the history of the daily activity records and room occupancy factors, would be the most applicable approach to deal with the above concerns.

Acknowledgements - The authors wish to express their gratitude for the financial support of the National Health Research Institute and National Science Council of Taiwan under Research Contract No. NHRI-DD01.86IX.RA501.P and NSC85-2331-B-010-054.

\section{References}

BEIR-III (1980) Advisory committee on biological effects of ionizing radiation - the effects on populations of exposure to low levels of ionizing radiation. National Academy Press. Washington, DC.

BEIR-V (1990) Advisory committee on biological effects of ionizing radiation - health effect of exposure to low levels of ionizing radiation. National Academy Press, Washington, DC.

Cardarelli II J. C., Hornung R., Robert H. and Chang W. P. (1997) Proposed model for estimating dose to inhabitants of Co-60 contaminated buildings. Health Physics 72, 351.

Cardis E., Gilbet E. S. and Carpenter L. et al. (1995) Effects of low doses and low dose rates of external ionizing radiation: Cancer mortality among nuclear industry workers in three countries. Radiation Research 142, 117.

Chang I. B. and Chang W. L. (1996) Exposure rates measurement of the buildings contamination with Co-60. Annual Research Report, Atomic Energy Council of Taiwan.

Chang W. P. and Kau J. (1993) Taiwan: Exposure to high doses of radiation. The Lancet 341, 750.

Degteva M. O., Kozheurov V. P. and Vorobyova M. I. (1994) General approach to dose reconstruction in the population expose as a result of the release of radioactive wastes into the Techa river. Science of the Total Environment 142, 49.

Degteva M. O., Kozheurov V. P., Burminstrov D. S., Vorobyova M. I., Valchuk V. V., Bougrov N. G. and Shishkina H. A. (1996) An approach to dose reconstruction for the rural population. Health Physics 71, 71.

Fisher H. L. and Snyder W. S. (1967) Distribution of dose in the body from a source of gamma rays distributed uniformly in an organ. Oak Ridge National Laboratory Report ORNL-4186, Oak Ridge, TN.

Gilbert E. S. (1991) Studies of workers exposed to low dose of external radiation. Occupational Medicine 6, 665.

Gilbert E. S., Fix J. J. and Baumgartner W. V. (1996) An approach to evaluation bias and uncertainty in estimates of external dose obtained from personal dosimeters. Health Physics 70, 336.

Goksu H. Y., Heide L. M., Bougrov N. G., Dalheimer A. R., Meckbach R. and Jacob P. (1996) Depthdose distribution in bricks determined by thermoluminescene and by Monte-Carlo calculation for external $\gamma$-dose reconstruction. Applied Radiation and Isotopes 47, 433.

Golikov V. Y. and Nikitin V. V. (1989) Estimation of the mean organ doses and the effective dose equivalent from rando phantom measurements. Health Physics 56, 111.

Grosswendt B. and Hohlfeld K. (1989) Effect of the irradiation geometry on dose-equivalent index. Health Physics 56, 657.

Haskell E. H. (1993) Accident dosimetry using environmental materials. Nuclear Tracks in Radiation Measurement 21, 87.

ICRP 60 (1991) Recommendations of the International Commission on Radiological Protection. ICRP Report 60, Pergamon Press, Oxford.

ICRU 39 (1985) Determination of dose equivalents resulting from external on external radiation source. ICRU Report 39. Bethesda, MD.

ICRU 43 (1988) Determination of dose equivalents resulting from external radiation sources, Part 2. ICRU Report 43, Bethesda, MD.

ICRU 47 (1992) Measurement of dose equivalents from external photon and electron radiation. ICRU Report 47, Bethesda, MD

Lubenau J. O. and Nussbaumer D. A. (1986) Radioactive contamination of manufactured products. Health Physics 51, 409.

Lubenau J. O. and Yusko J. G. (1995) Radioactive materials in recycled metals. Health Physics 68, 440.

Marley M. (1993) Radiative steel in Taiwan homes: some regulators could lose jobs. American Metals Market 14, 1.

Maruyama T., Kumamoto Y. and Noda Y. (1988) Reassessment of gamma dose from the atomic bombs in Hiroshima and Nagasaki. Radiation Research 113, 1.

Rosenstein M. (1982) Dose equivalent factors for human organs and tissues from external radiation. Applied Radiation and Isotopes 33, 1051.

Schultz F. W. and Zoetelief J. (1996) Organ and effective dose in the male phantom Adam exposed in AP direction to broad unidirectional beams of monoenergetic electrons. Health Physics 70, 498.

Xu X. G., Reece W. D. and Poston J. W. (1995) A study of the angular dependence problem in effective dose equivalent assessment. Health Physics 68, 214. 